\title{
INTERRUPTION OF MEDIUM-VOLTAGE DIRECT-CURRENTS BY SEPARATION OF CONTACT ELEMENTS IN MINERAL OIL USING AN ULTRA FAST ELECTRO-MAGNETIC ACTUATOR
}

\author{
S. JugelT*, C. LeU \\ Research Unit High-Voltage Technologies, Technische Universität Ilmenau, Gustav-Kirchhoff-Straße 1, 98693, \\ Ilmenau, Germany \\ * stefan.jugelt@tu-ilmenau.de
}

\begin{abstract}
The increasing usage of medium-voltage direct-current in upcoming electrical energy grid topologies requests novel solutions for MVDC switching. The interruption of direct-currents is accomplished by enforcing a current zero crossing by adequate means and preventing reignition due to the recovering dc voltage. This paper evaluates the rapid separation of the contact elements in mineral oil leading to a liquid flow around the contact elements and the switching arc. The energy turnover of dielectric liquids interacting with an electric arc is considerably higher leading to heavily increased arc voltages compared to dielectric gases. This paper confirms results of earlier publications and carries them further towards a possible usage in an MVDC switching or protection device. Thus a contact arrangement surrounded by mineral oil in combination with an ultra fast electro-magnetic actuator is introduced and performed measurements are discussed.
\end{abstract}

Keywords: dc interruption, bulk oil, dc switching arc; mvdc; mvdc protection device.

\section{Introduction}

The availability of high voltage power electronics leads to new concepts of HVDC and MVDC electrical energy grid topologies these days. But the usage of high and medium voltage direct currents imposes new difficulties regarding current interruption. With direct currents a current zero crossing does not naturally appear hence AC switchgear technologies are no capable of high and medium voltage current interruption. To overcome the lack of a current zero crossing three main principles are known [1]. Divergent oscillation (1) and current injection (2) utilize synthetic resonant networks in parallel to an ultra fast disconnecter (1) or main circuit breaker (2). The third principle, counter voltage (3), requires an arc (or semiconductor circuits) to build up a voltage drop along the current path higher than the driving voltage thereby forcing the current to zero. In this paper an approach to build up a high arc voltage conducting a contact separation in mineral oil as proposed in [2] is shown. The mechanical force and contact velocity needed is provided by an ultra fast electro-magnetic actuator.

\section{Test and measurement setup}

\subsection{Test setup}

To accomplish a fast contact separation in mineral oil a contact configuration within an extinguishing chamber as shown in figure 1 is used. This extinguishing chamber (1) is filled with mineral oil of type Shell Diala S4 ZX-I. The contact separation is accomplished by pushing (3) on the moving contact (4) by the electro-magnetic actuator (2) (see section 2.2).

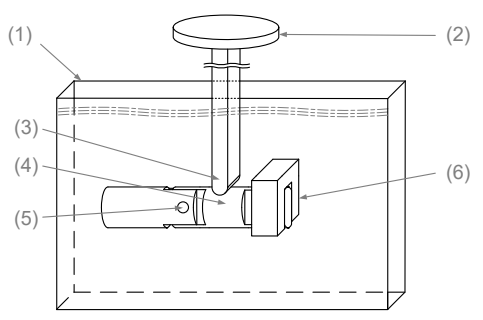

Figure 1. Contact configuration in the mineral oil filled extinguishing chamber with electro-magnetic actuator.

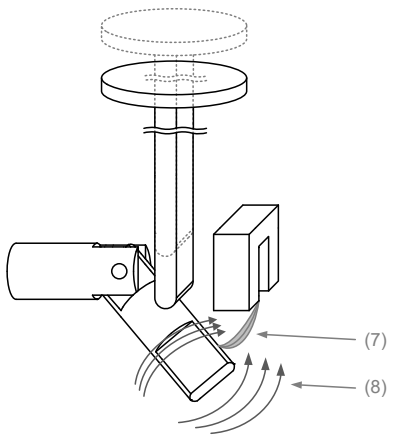

Figure 2. Electric arc (7) and liquid flow (8) during movement.

The moving contact (4) pivots around the pivot point (5) from $0^{\circ}$ (contact closed) to $-90^{\circ}$. Physical contact separation from the fixed contact (6) does occur $7 \mathrm{~mm}$ in to the mechanical movement of the moving contact (4) and pivoting ends $49 \mathrm{~mm}$ into the movement of the electro-magnetic actuator. After contact separation an electric arc (7) originates. Due to pivoting this contact (4) a liquid flow (8) of mineral 
oil on the tip of the contact (4) does occur, see figure 2, draining power from the electric arc.

\subsection{Electro-magnetic actuator}

It is known from [2] that contact separation or arc elongation in mineral oil needs to be very rapid in order to generate high arc voltages. Previously propellant powered drives were used to achieve these high velocities. Disadvantageous to propellent drives are there properties to be single use and there low velocity after ignition. Hence an ultra fast electro-magnetic actuator driven by a capacitor discharge is used here. These types of drives are also proposed for usage in ultra fast vacuum circuit-breakers [3].

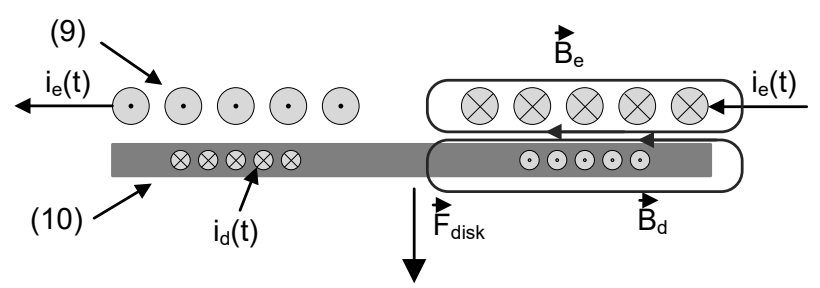

Figure 3. Schematic illustration of Thomson coil actuator.

A schematic illustration of such a ultra fast electromagnetic actuator is given in figure 3 . For the purpose of the conducted experiments only unifilar windings were used for the flat coil (9), in this shape often called THOMSON coil. The current $i_{\mathrm{e}}(t)(1)$ induces a voltage into the aluminium disk (10) resulting in eddy currents opposing the magnetic field of the Thomson coil (9).

$$
\begin{aligned}
& L_{B_{\mathrm{e}}} \cdot \frac{\mathrm{d} i_{\mathrm{e}}(t)}{\mathrm{d} t}-\frac{\mathrm{d} M_{B_{\mathrm{e}}, B_{\mathrm{d}}}(x(t)) \cdot i_{\mathrm{d}}(t)}{\mathrm{d} t}= \\
& \quad \int C_{0} \cdot i_{\mathrm{e}}(t) \mathrm{d} t-L_{\mathrm{par}} \cdot \frac{\mathrm{d} i_{\mathrm{e}}(t)}{\mathrm{d} t}-R_{\mathrm{par}} \cdot i_{\mathrm{e}}(t)
\end{aligned}
$$

$(x(t)$ - Displacement of the disk (10))

And the resulting force accelerating disk (10) is proportionate

$$
\begin{aligned}
\vec{F}_{\text {disk }}= & \iint \vec{J}_{\mathrm{d}}(x(t)) \mathrm{d} \vec{A}_{\text {disk }} \\
& \left(\vec{d}(t) \times \mu \frac{\partial}{\partial \vec{x}} \iint \vec{J}_{\mathrm{e}}(x(t)) \mathrm{d} \vec{A}_{\text {wire }}\right) \\
F_{\text {disk }} \sim & i_{\mathrm{e}}(t) \cdot i_{\mathrm{d}}(t)
\end{aligned}
$$

So with variation of load voltage $U_{\mathrm{TD}}$ and/or capacitance $C_{0}$ the speed of contact separation can be controlled.

\subsection{Electrical test circuit}

The conducted measurements were carried out using the electrical test circuit shown in figure 4.

A low inductance resistor damped capacitor discharge provides the energy against which the arc voltage is build up. This capacitor discharge is initiated

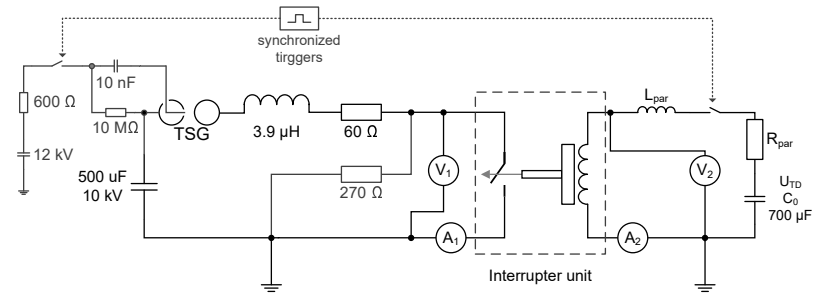

Figure 4. Electrical test circuit as used in measurements $\left(\tau_{\text {discharge }}=30 \mathrm{~ms}\right)$

left: low inductance medium voltage dc circuit right: electrical circuit of the electro-magnetic actuator.

by a spark gap (TSG) triggered by a controlled voltage exaltation on the third (pin) electrode. This voltage exaltation is synchronized to the excitation of the electro-magnetic actuator by timer/counter blocks of a micro controller. Additionally the velocity of the electro-magnetic actuator is measured in direction of the movement using a laser triangulation sensor (40 kHz, measuring range: $50 \mathrm{~mm}$, resolution: $50 \mu \mathrm{m}$ ).

\section{Theoretical considerations on the decomposition of mineral oil}

Electric arcs in dielectric liquids generate a gaseous phase around them [4]. As for highly molecular liquids such as the used Shell Diala S4 ZX-I due to the high arc temperature dissociation leading up to the atomic level does occur. Following diffusion into colder areas of the gaseous phase partial recombination does occur releasing part of the bond-dissociation energy heating up the gaseous phase. Furthermore the chemical dissociation reaction can be described by

$$
\mathrm{C}_{18} \mathrm{H}_{38} \rightarrow 10 \mathrm{H}_{2}+5 \mathrm{C}_{2} \mathrm{H}_{2}+2 \mathrm{CH}_{4}+6 \mathrm{C}
$$

where only the shorted possible alkane $\left(\mathrm{C}_{n} \mathrm{H}_{2 n+2}\right)$ [5] is taken into consideration because of lower bonddissociation energy. Which is defined by

$$
\begin{array}{rl}
\mathrm{H}-\mathrm{H} & 103.4 \mathrm{kcal} / \mathrm{mole} \\
\mathrm{C}-\mathrm{C} & 58.6 \mathrm{kcal} / \mathrm{mole} \\
\mathrm{C} \equiv \mathrm{C} & 123 \mathrm{kcal} / \mathrm{mole} \\
\mathrm{C}-\mathrm{H} & 87.3 \mathrm{kcal} / \mathrm{mole} \\
\mathrm{C}_{\text {gaseous }} \rightarrow \mathrm{C}_{\text {solid }} & 124.3 \mathrm{kcal} / \mathrm{mole}
\end{array}
$$

as per [6]. So to dissolve a hydrocarbon molecule $4314 \mathrm{kcal} / \mathrm{mole}$ of energy is needed. Likewise $3967 \mathrm{kcal} / \mathrm{mole}$ do get converted during recombination. So for the chemical reaction

$$
347 \mathrm{kcal} / \mathrm{mole}
$$

of energy needs to be supplied. With a molecular weight of $254 \mathrm{~g} /$ mole $\left(\mathrm{C}_{18} \mathrm{H}_{38}\right)$ the chemical reaction energy is

$$
E_{\text {decomp }}=1.37 \mathrm{kcal} / \mathrm{g} \approx 5.7 \mathrm{~kJ} / \mathrm{g}
$$


Additionally for the electric arc to sustain within the gaseous phase energy is needed to heat up the products of decomposition. For heated highly pressurized gases of this matter the specific heat is dominated by the concentration of $\mathrm{H}_{2}$ [7]. Based on the enthalpy of high temperature pressurized $\mathrm{H}_{2}$ which according to [8] can be estimated to $31 \mathrm{~kJ} / \mathrm{g}_{\mathrm{H}_{2}}$ and the mass faction of $\mathrm{H}_{2}$ in $\mathrm{C}_{18} \mathrm{H}_{38}$ of $0.18 / \mathrm{g}_{\mathrm{C}_{18} \mathrm{H}_{38}}$ an additional energy of

$$
\mathrm{E}_{\text {heat }} \approx 5.5 \mathrm{~kJ} / \mathrm{g}
$$

is required to heat up the products of decomposition by the electric arc.

\section{Measurements}

With the contact configuration described in section 2.1 measurements were carry out varying the velocity of the electro-magnetic actuator hence varying the speed of contact separation. The results can be grouped into two voltage/current-time characteristics.

For the purpose of this paper the first voltage/current-time characteristic is treated as a successful current interruption. An example of a measured successful current interruption is given in figure 5 .

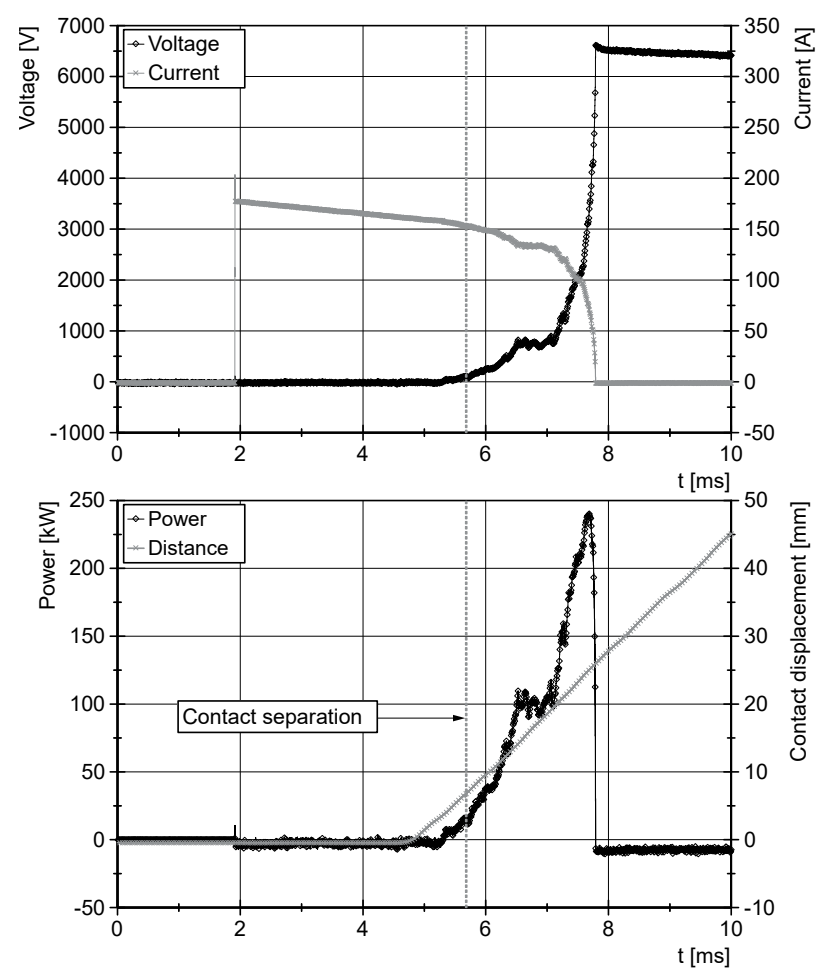

Figure 5. Top: arc voltage and current for a current interruption at $v_{\text {contact }}=9.0 \mathrm{~m} / \mathrm{s}$;

bottom: power output of the electric arc and measured displacement of the moving contact.

After contact separation at $\sim 5.8 \mathrm{~ms}$ the arc voltage increases nearly exponentially until it reaches the capacitor discharge voltage. Contrary to that the current decreases exponentially following the voltage rise until it reaches zero and current interruption is accomplished within $1.8 \mathrm{~ms}$. With the applied velocity of the moving contact of $9.0 \mathrm{~m} / \mathrm{s}$ the displacement at current interruption is just $18 \mathrm{~mm}$ hence the contact is not fully pivoted in this case. Due to the continuing pivot movement and the very high dielectric strength of the mineral oil during all measurements no rearcing process could be observed. For the case of the current interruption of figure 5 the power output of the electric arc reaches $240 \mathrm{~kW}_{\text {peak }}$ immediately before the current declines to zero, inducing $210 \mathrm{~J}$ of energy into the liquid.

Opposed to the voltage/current-time characteristic of figure 5 , under the same constrains of capacitor load voltage, discharge current and trigger delay of the electro-magnetic actuator but lower velocity $(7.2 \mathrm{~m} / \mathrm{s})$ an example of a non successful current interruption is given in figure 6 .

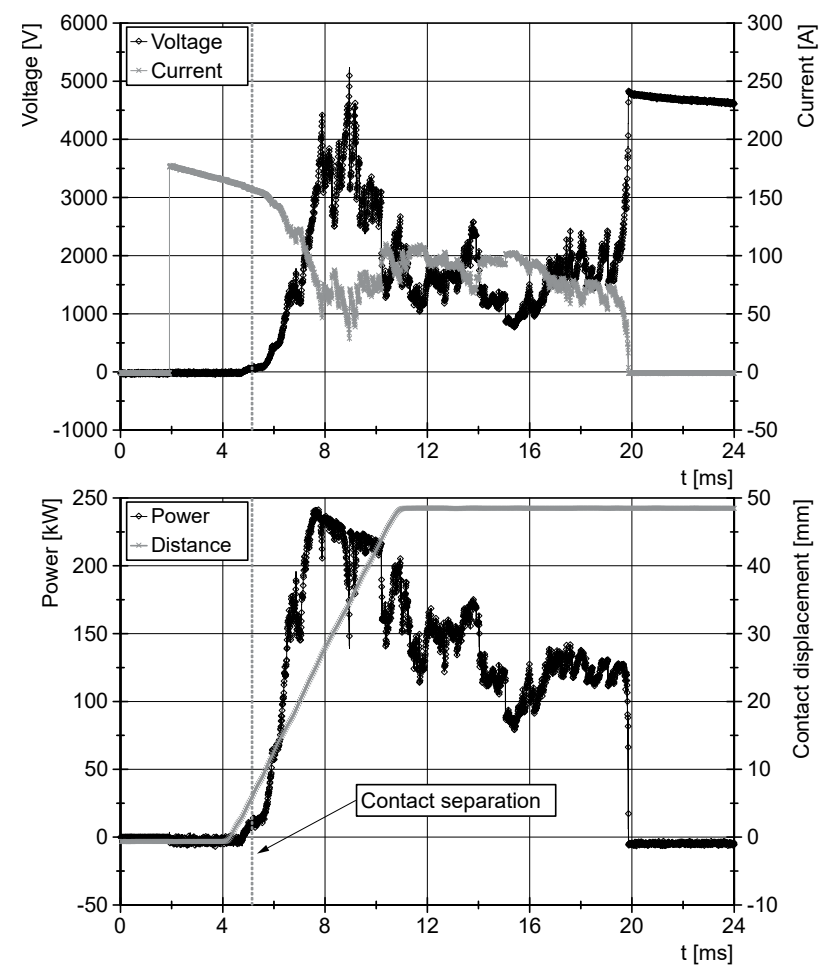

Figure 6. Top: arc voltage and current for a current interruption at $v_{\text {contact }}=7.2 \mathrm{~m} / \mathrm{s}$;

bottom: power output of the electric arc and measured displacement of the moving contact.

After contact separation at $5.8 \mathrm{~ms}$ the arc voltage at first rises exponentially to but the current does not follow in the same manner as before. At $7.8 \mathrm{~ms}$ (moment of current interruption of fig. 5) the power output of the arc peaks at $245 \mathrm{~kW}$ but due to the lower velocity of the moving contact the induced energy at $1.8 \mathrm{~ms}$ after contact separation is already $320 \mathrm{~J}$ hence an increased volume of mineral oil is decomposed (cf. (11)). The major gaseous phase provides sufficient volume for the arc to further heat up the decomposition products (cf. (12)) and the increasing gas volume leads to an electric arc surrounded only by gaseous $\mathrm{H}_{2}$. Current interruption is still accomplished 
$14.2 \mathrm{~ms}$ after contact separation due to the decreasing capacitor voltage and the continuing pivot movement.

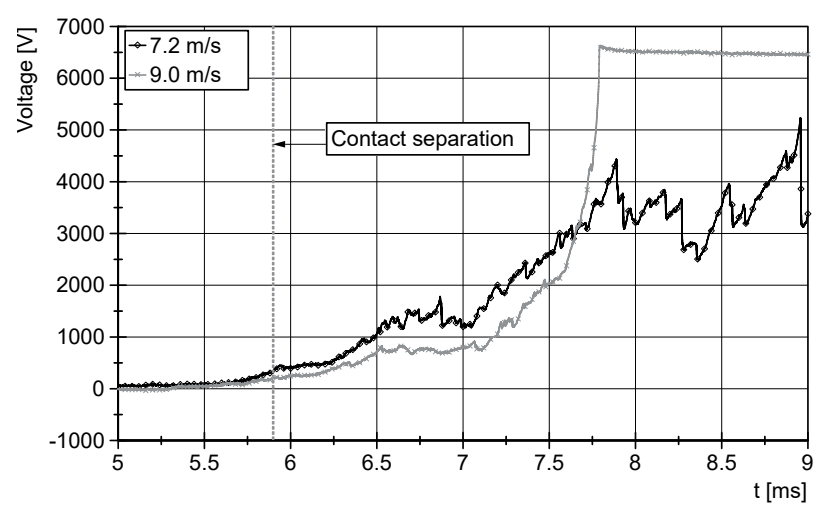

Figure 7. Arc voltage immediately after contact separation.

As seen in figure 7 the arc voltage increases higher immediately after contact separation in case of a lower velocity. This is due to the low turbulence flow of the mineral oil in this case. The electric arc is able to decompose more (stationary) liquid hence the higher arc voltage but the generated gas can not removed from the arcing zone sufficiently. For high velocities the liquid stream can remove more gas but arc voltage is limited by the distorted surface of the phase boundary.

To further verify this behaviour the accumulated energy of the electric arc induced into the liquid $1 \mathrm{~ms}$ after contact separation is evaluated for the measured velocities, see figure 8.

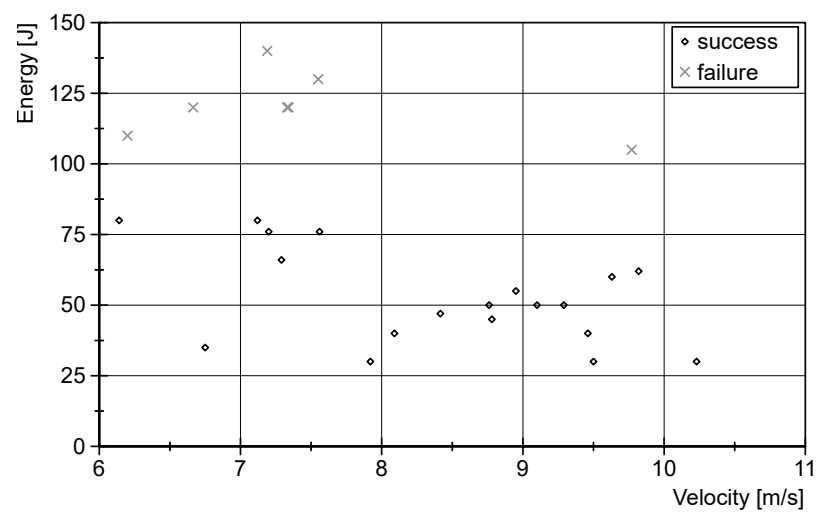

Figure 8. Accumulated arc energy $1 \mathrm{~ms}$ after contact separation.

The results reveal that a successful current interruption (as seen in figure 5) could only be accomplished when a low amount of energy is induced into the liquid by the electric arc within the first moments after contact separation. If however the energy turnover of the electric arc immediately after contact separation is rather high, current interruption will fail afterwards. As for dependency of the velocity, due to slight variations of the electro-magnetic actuator trigger delay, current interruptions for low $(\sim 6-8 \mathrm{~m} / \mathrm{s})$ velocities are observed too, because voltage and current of the charged capacitor are decreased further leading to lower power output of the arc for a increased trigger delay. Futher it can be seen in figure 8 that the induced energy $1 \mathrm{~ms}$ after contact separation decreases with increasing velocity. It should be assumed that due to the turbulent flow for high velocities the electric arc is quenched more leading to a increased current density but less radiated heat hence lower energy induced into the liquid.

\section{Conclusions}

Based on the measurements shown before multiple conclusions regarding medium voltage direct current interruption utilizing the counter voltage principle can be drawn.

1. Ultra fast electro-magnetic actuators, which are of huge interest for UFDs and vacuum circuit breakers, are very well suited for medium voltage directcurrent interruption within dielectric liquids like mineral oils.

2. Mechanical contact separation for MVDC interruption in dielectric liquids needs to be very fast to avoid excessive generation of gaseous decomposition products.

3. Utilizing appropriative velocities low current MVDC interruption can be accomplished with a contracted electric arc avoiding intermediate current rise and voltage drop within very short periods of time (for mechanical current interruption).

4. For high current interruption the previously shown principle of high speed contact separation may not be sufficient because of the power dissipation of the electric arc $\left(P \sim I^{2}\right)$ hence a forced liquid flow is needed.

\section{References}

[1] V. Lenz. DC current breaking solutions in HVDC applications. Master's thesis, High Voltage Laboratories, ETH Zurich, 2015.

[2] H. Ann. Untersuchungen über die Erzeugung sehr hoher Lichtbogenspannungen unter Flüssigkeiten. $\mathrm{PhD}$ thesis, TH Braunschweig, 1966.

[3] A. Bissal. Modeling and Verification of Ultra-Fast Electro-Mechanical Actuators for HVDC Breakers. PhD thesis, KTH, Electromagnetic Engineering, 2015. QC 20150422.

[4] F. Kesselring. Das Schaltproblem der Hochspannungstechnik. Archiv für Elektrotechnik, 35(3):155-184, Mar 1941.

[5] Shell Deutschland Oil GmbH. Sicherheitsdatenblatt Shell Diala S4 ZX-I. Technical report, 2018. 
[6] R. Heizmann. Abschaltversuche mit verschiedenen Löschflüssigkeiten und die Zusammensetzung der entstehenden Schaltergase. PhD thesis, ETH Zurich, 1957.

[7] R. Kuthe. Temperaturen und Zusammensetzung eines aus Kohlenstoff und Wasserstoff entstandenen hocherhitzten Gases in Abhängigkeit vom Druck. PhD thesis, TH Braunschweig, 1961.

[8] F. Burhorn et al. Plasmazusammensetzung, Plasmadichte, Enthalpie und spezifische Wärme von Wasserstoff und Wasser bei 1,3,10 und $30 \mathrm{~atm}$ im Temperaturbereich zwischen 1000 und $30000 \mathrm{~K}$. Zeitschrift für Physikalische Chemie, 215, 1960. doi:10.1515/zpch-1960-21528. 